\title{
Il genere biografico degli umanisti e la ricezione nella Penisola Iberica nel Quattrocento: appunti per una revisione*
}

\author{
Susanna Allés Torrent \\ Institució Milà i Fontanals - CSIC \\ susannalles@imf.csic.es
}

\begin{abstract}
La ripresa del genere biografico sotto i parametri degli autori greci e latini fu durante il Quattrocento italiano un fenomeno di vasta portata letteraria e culturale la cui onda espansiva arrivò negli stessi anni nella Penisola Iberica. Alcuni capitoli di questa ricezione sono da collegarsi direttamente al ricupero di Plutarco e le traduzioni latine quattrocentesche delle Vite parallele; un gran numero di queste versioni furono edite per la prima volta verso il 1470, posteriormente una ristampa dell'anno $1478 \mathrm{fu}$ integralmente tradotta in lingua spagnola; questo fatto implicò un allargamento considerevole di opuscoli biografici in territorio ispanico, non sempre presi con la dovuta considerazione.
\end{abstract}

Palabras clave: biografie; Quattrocento; traduzioni; Alfonso de Palencia.

\begin{abstract}
The recovery of the biographical genre in the framework of the Greek and Latin authors was a phenomenon during the Italian Quattrocento of great literary and cultural reach whose expansive wave reached the Iberian Peninsula in the same period. Some chapters of this reception are to be connected directly to the resurgence of Plutarch and the Quattrocento Latin translations of the Vite parallele. A large number of these versions were published for the first time around 1470 and a 1478 reprint was later fully translated into Spanish; this resurgence involved a considerable increase of biographical booklets in Hispanic territory, not always taken with due consideration.
\end{abstract}

Keywords: Biographies; Quattrocento; translations; Alfonso de Palencia.

* Premetto che per le sigle delle biblioteche si useranno: BNE, per la Biblioteca Nacional de España (Madrid), BNCF, per la Biblioteca Nazionale Centrale di Firenze. Ugualmente, sarà abbreviato in «Progetto Boscán», il PROYECTO BOSCÁN: Catálogo de las traducciones españolas de obras italianas (hasta 1939) [on line]. <http://www.ub.edu/boscan> [ultima consulta: 03-04-2013]. 


\section{Contesto generale: il genere biografico in Italia e alcune tracce della sua ricezione}

Durante il Quattrocento il genere biografico riscosse un grande successo e fu quello che probabilmente meglio rappresentò, all'interno del pensiero umanistico, l'idea fondamentale della centralità dell'uomo. Ad ogni modo, in qualità di genere letterario non ebbe un carattere monolitico e sarebbe una questione molto complessa misurare in che grado si intrecciarono tradizioni diverse: ingredienti e cliché appartenenti alle leggende agiografiche; elementi delle introduzioni ad autori celebri (i ben noti accessus ad autores); motivi ispirati alla tradizione epica; o modelli biografici di matrice classica.

In uno studio sullo sviluppo della scrittura biografica, M. McLaughlin ${ }^{1}$ evidenziò una triplice tipologia testuale: da una parte, le collezioni o gallerie di personaggi illustri, iscritti nella tradizione dei De viris illustribus; da un'altra, le biografie individuali, molte volte ritratti a mo' di panegirico di singole personalità; infine, le autobiografie che, sulla scia introspettiva del Secretum e della Lettera ai posteri del Petrarca, trovarono la più compiuta espressione tanto nell'autobiografia dell'Alberti (c. 1460) quanto soprattutto in quelle rinomate di Benvenuto Cellini (Vita, scritta tra 1558-1566, ma pubblicata nel 1728) e Girolamo Cardano (De vita propria, 1575).

In questa sede ci interessa il secondo grande gruppo, costituito dalle biografie individuali di personaggi antichi e moderni, per lo più panegirici di mecenati — nel caso dei governanti-, ma anche di artisti e scrittori. I modelli sono da rintracciare nei paradigmi classici di Plutarco, Diogene Laerzio, e quasi sicuramente nelle vite della tarda antichità di poeti come Virgilio, al quale —com'è risaputo- venne dedicata una larga serie di Vite vergiliane. Senz'altro, il grande precedente in questo caso è Boccaccio, il quale scrisse il De vita et moribus Francisci Petracchi (1348), in lingua latina, e il Trattatello in laude di Dante (1351-53, riscritto nel 1363). Queste due ultime opere interessano particolarmente perché, come dimostrano Gómez Moreno e Jiménez Calvente, ${ }^{2}$ circolarono nell'ambiente del Marchese di Santillana. Difatti la vita di Dante fu oggetto di molteplici biografie dove spiccano autori come Filippo e Giovanni Villani, Giannozzo Manetti e, soprattutto, Leonardo Bruni. Di quest'ultima conserviamo una traduzione anonima in lingua castigliana realizzata verso il 1450 e indirizzata a Íñigo López de Mendoza, Marchese di Santillana, sotto il titolo di La vida et estudios et costumbres de Dante et de micer Francisco Petrarca, oggi manoscritto BNE, ms. $10171 .^{3}$

1. Martin McLaughlin, «Biography and Autobiography in the Italian Renaissance», in Peter France-William St. Clair (a cura di), Mapping Lives: the uses of biography, Oxford: Oxford University Press for the British Academy, 2002, p. 37-65.

2. Ángel Gómez Moreno-Teresa Jiménez Calvente, «De Dante y otras vite», Cuadernos de Filología Italiana, n. extraordinario, 2001, p. 373-392.

3. Cfr. Giuseppe Mazzocchi-Paolo Pintacuda, «La versione castigliana quattrocentesca delle 'Vite di Dante e del Petrarca' di Leonardo Bruni», in Luisa Rotondi Secchi Tarugi (a cura di), Rapporti e scambi tra umanesimo italiano ed umanesimo europeo, Milano: Nuovi 
Non mancarono naturalmente biografie di re e governanti, con un'intenzione più laudativa che critica, in cui spicca la figura di Alfonso il Magnanimo. Non posso, per questioni di spazio, soffermarmi su questi lavori, ma mi limiterò a ricordare che il De dictis et factis Alphonsi regis di Antonio Beccadelli, il Panormita (1394-1471), generò in Spagna una ricca serie di traduzioni a cui diede avvio Jordi de Centelles verso l'anno 1481, con Lo llibre dels fets e dits del gran rey Alfonso; posteriormente, durante il Cinquecento spagnolo continuarono le versioni grazie a Juan de Molina (1527) e, in modo parziale, a Fortunio García de Ercilla (1530-1550) e Antonio Rodríguez Dávalos (1554); il testo - concretamente il libro III, capitolo 36 - fu rivisitato da Pere Miquel Carbonell nell'opera intitolata Sobre una singular visitació (1546). Alcuni testi di questo genere, dedicati a una singola personalità, finirono per diventare, per il loro carattere chiaramente encomiastico, piccole creazioni epiche, com'è il caso di Pier Candido Decembrio, autore di una biografia del duca di Milano, Filippo Maria Visconti, composta nel 1447 e ispirata ai modelli svetoniani; sempre allo stesso filone sono ascrivibili le sue biografie di Francesco Sforza e quella di Ercole d'Este, scritta questa dopo essere stato assunto, nel 1471, nel ducato di Ferrara. ${ }^{4}$

Il modus faciendi proprio degli umanisti si estese fino alle biografie di pontefici, lontane però ormai dai registri medievali degli atti dei papi. ${ }^{5} \mathrm{Ne}$ sono un esempio le biografie scritte da Bartolomeo Sacchi, il Platina (1421-1481) che, nella sua storia dei pontefici romani (Liber de vita Christi ac omnium pontificum), giunge fino al suo protettore Sisto IV.

$\mathrm{Ci}$ fu, infine, un gruppo di biografie consacrate a saggi antichi, scrittori e filosofi, di cui ci occuperemo nelle pagine successive di quest'articolo e il cui impatto nella Penisola Iberica è in parte da ricostruire. Si trattò di un gruppo di biografie più direttamente ispirate agli autori classici: Plutarco, Svetonio, Valerio Massimo e Diogene Laerzio vengono scelti non solo come modelli ma anche come fonti primarie. Giannozzo Manetti, come poi molti altri faranno, nella sua dedica delle vite di Seneca e Socrate a Nuño de Guzmán e poi ad Alfonso il Magnanimo sottolinea esplicitamente come la sua ispirazione provenga da Plutarco. ${ }^{6}$

orizzonti, 2001, p. 439-489; Lorenzo BARTOLI, «La versione castigliana delle 'Vite di Dante e del Petrarca' e la 'controversia alphonsiana': osservazioni filologiche sui rapporti fra Bruni e la Spagna in epoca conciliare», in María N. Muñiz Muñiz (a cura di), La Traduzione della Letteratura Italiana in Spagna (1300-1939). Traduzione e tradizione del testo: dalla filologia all'informatica, Firenze: Franco Cesati Editore, 2007, p. 175-182.

4. Questa tipologia di testi, riconducibili al panegirico, ebbe il suo corrispettivo nelle figure dei Re Cattolici, come segnalato da Ángel Gómez Moreno, España y la Italia de los humanistas, Madrid: Gredos, 1994, p. 235-236.

5. Cfr. Massimo Miglio, Storiografia pontificia del Quattrocento, Bologna: Patron Editore, 1975.

6. Alla fine della prefazione a Nuño de Guzmán, Manetti dichiara apertamente di essersi ispirato a Plutarco: «In qua quidem re Plutarchum pre ceteris imitati sumus, qui quosdam clarissimos viros cum Grecos tum Latinos invicem comparavit et egregias 
Di sicuro il caso più fecondo nel contesto delle scritture biografiche è appunto quello di Giannozzo Manetti, autore di vite di pontefici (De vita ac gestis Nicolai quinti summi pontificis) e di personaggi politici antichi e moderni come risulta dalla Vita Philippo regis Macedonum, dalla Vita Alphonsi regis Aragonum, o dal Liber de illustribus longevis, del quale Nuño de Guzmán portò una copia in Spagna. Per quanto riguarda le biografie di scrittori, compilò verso il 1440 le vite parallele di Socrate e Seneca (Vita Socratis et Senecae) che, pur essendo dedicate a un personaggio spagnolo, Nuño de Guzmán, non furono oggetto di alcuna traduzione in lingua castigliana. Quindi, intorno agli stessi anni, redasse le Vitae Dantis et Petrarchae ac Boccaccii. ${ }^{7}$

Interessante continua ad essere il lavoro svolto da Pier Candido Decembrio, uno degli autori con maggior influenza nella Penisola Iberica. Su richiesta di Alfonso de Cartagena dedicò al re Giovanni II di Castiglia la traduzione dei libri I-IV e X dell' 'liade e anche una Vita Homeri, ambedue conservate, tra altri opuscoli dello stesso autore, nel ms. Add 21245 della British Library, nel quale si conserva peraltro anche una traduzione spagnola, incaricata dal proprio Marchese di Santillana e curata da Saquero - González. ${ }^{8}$

Infine, per completare la visione d'insieme di questo gruppo di biografie di personaggi antichi giunte in traduzione in Spagna, vorrei a questo punto sottolineare le versioni approntate da Alfonso de Palencia che, non sempre in modo deliberato contribuì ad arricchire questo repertorio biografico.

\section{Scelte editoriali dalle conseguenze imprevedibili: le Vite parallele e altre biografie}

In altre sedi abbiamo seguito le vicende e il ruolo centrale svolto dalle traduzioni latine delle Vite parallele di Plutarco, raccolte e stampate sotto la guida di Giovannatonio Campano nel $1470 .{ }^{9}$ Una terza edizione di questo corpus,

quasdam comparatorum hominum Vitas grecis litteris mandavit, atque exinde ingens et memorandum opus absolvit, quod Greci Parallela inscripserunt.» Vita Socratis et Senecae, Alfonso De Petris (a cura di), Firenze: L.S. Olschki, 1979, p. 24.

7. Il «progetto Boscán» indica come uniche traduzione di Manetti, l'Orazione a Gismondo Pandolfo de'Malatesta, portata a termine da Nuño de Guzmán verso il 1458, e un'altra anonima più tardiva, forse da collocarsi tra il 1470 e il 1490; cfr. Jeremy N. H. LaWRANCE, Un episodio del protohumanismo español. Tres opúsculos de Nuño de Guzmán y Giannozzo Manetti, Salamanca: Biblioteca Española del siglo xv, 1989, p. 193-228.

8. Pilar Saquero SuÁrez-Somonte - Tomás GonZÁlez Rolán, «Sobre la presencia en España de la versión latina de la Ilíada de Pier Candido Decembrio. Edición de la Vita Homeri y de su traducción castellana», Cuadernos de Filología Clásica, n. 21, 1988, p. 319-344, concretamente p. 319-320 (e a p. 324 l'elenco dei manoscritti conservati); Alfred MorelFatio, «Les deux 'Omero' castillans», Romania, n. 25, 1896, p. 111-120 (pubblicato anche in Études sur l'Espagne, 4. a serie, Paris, 1925, p. 88-103); Pedro M. Cátedra, «Sobre la biblioteca del Marqués de Santillana: La Iliada y Pier Candido Decembrio», Hispanic Review, vol. 51, n. 1 (Winter), 1983, p. 23-28;

9. Susanna Allés Torrent, «Alfonso de Palencia y la traducción de las Vidas de Plutarco (nuevos datos en torno al texto de partida)», Cuadernos de Filología Clásica. Estudios Latinos, 
stampata a Venezia per i tipi di Jenson nel 1478, arrivò in Spagna e fu tradotta integralmente dal cronista dei Re Cattolici, Alfonso de Palencia. ${ }^{10}$ L'inserzione di numerosi opuscoli biografici e storici in queste edizioni significò la diffusione non solo delle biografie plutarchee che erano state tradotte dal greco al latino da vari illustri umanisti, ma anche di tutta un'altra serie di lavori. Non si tratta di aggiunte arbitrarie o irrilevanti rispetto agli autentici plutarchei, poiché sono giustificati appunto dal loro carattere storiografico e biografico. Ad ogni modo, l'inclusione di questi testi ebbe delle conseguenze che Campano non aveva previsto perché significò la loro divulgazione in Italia e in Europa, ed anche la traduzione in altre lingue volgari, tra cui lo spagnolo.

Passerò quindi in rassegna queste opere biografiche, distinguendo, da un lato, le traduzioni di opere latine antiche e traduzioni indirette di opere greche e, dall'altro, le opere biografiche originali scritte da umanisti italiani. Lascerò, invece, da parte un lavoro di chiara natura storiografica presente nella raccolta, cioè il Breviarium rerum gestarum populi Romani di Rufo Festo $(\dagger c .379) .{ }^{11}$

\subsection{Scritti biografici di autori latini e traduzioni latine di testi biografi greci}

\section{La vita di Attico di Cornelio Nepote}

La vulgata campaniana conteneva due testi biografici di autori latini: uno di essi, la vita di Virgilio dovuta a Donato (s. III), purtroppo non passò alle edizioni posteriori e perciò non giunse in traduzione nell' arco del s. XV in Spagna; l'altra, invece, è la vita di Pomponio Attico scritta da Cornelio Nepote (c. 99 aC. - c. $24 \mathrm{aC}$.) ed inserita nel suo De viris illustribus, la cui editio princeps apparse nel $1471^{12}$. La biografia di Attico, che molto probabilmente circolava verso gli anni trenta, fu inclusa dal Campano perché rientrava nella linea della

28, n. 2, 2008, p. 99-124; cfr. inoltre i lavori ormai classici: Vito R. Giustiniani, «Sulle traduzioni latine delle Vite di Plutarco nel Quattrocento», Rinascimento, ser. II, n. 1, 1961, p. 3-62; Marianne PADE, The Reception of Plutarch's Live in Fifteenth-Century Italy, Copenhagen: Museum Tusculanum - University of Copenhagen, 2007.

10. Le edizioni a cui mi riferirò d'ora in avanti sono: Plutarco, Vitae illistrium virorum, J.A. Campano (ed.), [Roma]: Ulrich Han (Uldaricus Gallus), [1470]; ID., Vitae illustrium viorum, Venezia: Nicolaus Jenson, 2 gennaio 1478; ID., [Vitae illustrium virorum], trad. Alfonso de Palencia, 2 vol., Sevilla: Cuatro compañeros alemanes, 2 luglio I49I.

11. Sexto Rufo Festo, The Breviarium of Festus. A Critical Edition with Historical Commentary, J.W. EADIE (a cura di), London: Athlone press, 1967; ID., Abrégé des hauts faits du peuple romain, M.P. Arnaud-Lindet (ed. e trad. a cura di), Paris: Les Belles Lettres, 1994. Per quanto riguarda il Breviario, Alfonso de Palencia lo collocò alla fine della sua traduzione e vi aggiunse questo preliminare: «Síguese la epístola de Ruffo embiada al emperador Valentiniano, de la dignidad real y consular y imperial y de la accessión del imperio romano; la qual epístola, puesta en fin de las vidas de Plutharco y de otras algunas, traduxo el cronista Alfonso de Palençia de latín en romançe» (f.345v).

12. Cornelio Nepote, Vitae imperatorum, sive De vita illustrium virorum, Venezia: Nicolaus Jenson, 8 marzo 1471; almeno altre tre furono pubblicate nel corso del XV secolo: una a Milano nel 1480, un'altra a Brescia e un'altra a Venezia nel 1498. 
sua raccolta, in cui si trovava una vita di Cicerone, interlocutore epistolare di Attico; ciò senza dimenticare l'ufficio di biografo per il quale era conosciuto lo stesso Nepote ed il fatto che era stato perfino utilizzato per la stesura delle biografie plutarchee.

Per quanto riguarda la traduzione spagnola di Palencia, dati interessanti emergono sin dal prologo. Dobbiamo premettere che l'edizione principe del 1470 offriva come titolo alla biografia: «Incipit Vita Pomponii Athici clarissimi viri e greco sermone in latinum per Cornelium Nepotem translata» (ed. 1470, f. 478); mentre quella del 1478 segnalava, come autore, il nome di Plutarco: «Pomponi Attici viri illustris vita ex Plutarcho graeco in latinum per Cornelium Nepotem versa» (ed. 1478, f. 443v). Ambedue indicavano che si trattava di una traduzione dal greco al latino fatta dallo stesso Nepote, ma solo la seconda indicava esplicitamente Plutarco in qualità d'autore, errore che Palencia rilevò, ma con cautela, come si vede dalle sue parole:

Plutarcho philósopho escrivió en griego la vida de Pomponio Áttico, varón ylustre. Y, segund pareçe, fallola escripta en latín por Cornelio Nepote pariente de Cicerón que fue antes mucho que Plutarcho. Y el cronista Alfonso de Palencia la traduxo en romançe castellano

Palencia, Plutarco, II, f.318r.

Palencia si sentì costretto a dare ulteriori spiegazioni: da una parte, ipotizza che il testo originale doveva essere in latino, e dall'altra, afferma che, a conti fatti, Cornelio Nepote è, cronologicamente, molto anteriore a Plutarco. Sembra chiaro che Palencia sapeva chi era Nepote, o almeno sapeva che si trattava di un autore vissuto molto prima di Plutarco; meno sicura sarebbe la risposta se ci chiedessimo che cosa conoscesse esattamente dell'opera di Nepote o che tipo di notizie avesse su di lui: rimane pertanto aperta la questione sulla diffusione che tale autore poté avere nella Spagna della fine del xv secolo.

\section{Isocrate e l'Evagora, tradotto da Guarino}

Un'altra delle traduzioni spagnole arrivata attraverso Alfonso de Palencia è quella dell'Evagora di Isocrate (Atene 436 - $338 \mathrm{aC}$ ), uno degli autori prediletti degli umanisti italiani e largamente tradotto durante il Quattrocento. ${ }^{13} \mathrm{Al}$ di là dall'attrazione culturale degli insegnamenti destinati ai principi (carattere chiaramente presente nell'A Nicocle e nell'Evagora) e della teorica rivalità con Demostene, le dimensioni ristrette degli scritti isocratici erano specialmente adeguati — come ha segnalato Gualdo Rosa— per «esercitarsi nella lingua greca, e per avere più rapidamente a portata di mano

13. Per la fortuna di Isocrate durante il s. xv, si vedano i lavori di Lucia GuAldo Rosa: «Le traduzioni latine dell' A Nicolcle di Isocrate nel Quattrocento", in J. IJsEwIJn-E. Kessler (a cura di), Acta Conventus Neo-latini Lovaniensis, Proceedings of the First International Congress of Neo-Latin Studies, Louvain 23-28 August 1971, [Leuven]: Leuven University Press, 1973, p. 275-303; ID., La fede nella paideia. Aspetti della fortuna di Isocrate nei secoli XV e XVI, Roma: Nella sede dell'Istituto, 1984. 
sempre nuove opere da inviare, precedute da una dedica d'occasione, a futuri probabili protettori». ${ }^{14}$

La traduzione dell'Evagora che entrò a far parte del corpus latino delle Vite parallele di Plutarco fu quella compiuta da Guarino Veronese verso il 1434. In precedenza, Guarino aveva già intrapreso altre traduzioni isocratiche; infatti, lo si può considerare uno dei maggiori diffusori dell'opera di Isocrate, in possesso di un numero molto considerevole di manoscritti, paragonabile soltanto a quello di Plutarco o Platone. ${ }^{15}$

L'Evagora, quindi, un'opera che fluttua tra l'orazione funebre e l'elogio encomiastico per il re di Salamina, fu tradotta in spagnolo attraverso la versione latina di Guarino. Il titolo che accompagna la versione di Palencia ci fornisce la prova che il traduttore la considerava opera di Plutarco:

Plutarcho philósopho escriuió en griego la vida del ylustre varón Euágoras. Boluiola en griego Guarino Veronés y el cronista Alfonso de Palençia la traduxo en romançe castellano.

Palencia, Plutarco, II, f.312v.

Non v'è nessuna divergenza tra queste parole e quelle che precedono le vite propriamente plutarchee. Per quanto riguarda le traduzioni romanze, non ne arriverà nessunaltra fino a quella italiana di Ludovico Domenichi del $1566 .{ }^{16}$

\section{Le Vite di Omero}

Dobbiamo ora riprendere la figura di Omero. Il fascino per il vate veniva da lontano e ormai Petrarca e Boccaccio ne avevano dato fede. Seguirono i passi del Petrarca Coluccio Salutati, Leonardo Bruni, Guarino Veronese, Lorenzo Valla o Angelo Poliziano, ricavandone una qualche traduzione. Accanto però ai due capolavori, si sviluppò una letteratura periomerica di opere apocrife e di biografie del vate. Un caso paradigmatico di quest'ultimo gruppo di letteratura, come abbiamo visto, fu la stesura da parte di Pier Candido Decembrio d'una Vita Homeri, tradotta pochi anni dopo in Spagna.

Ebbene, allo studio di Omero nella Spagna del XV si deve aggiungere una traduzione parziale dovuta sempre ad Alfonso de Palencia nella sua versione delle Vite parallele (1491). Mi riferisco al De Homero di un certo PseudoPlutarco, tradotto dal fiorentino Pellegrino degli Agli (Firenze 1440 - Roma c. 1469), benché per molti anni fosse stata ritenuta opera di Guarino Veronese. ${ }^{17}$ Campano aggiunse una seconda biografia attribuita a Erodoto su origini,

14. Gualdo Rosa, «Le traduzioni latine ...». cit., p. 275-303.

15. Per le traduzioni di opere isocratiche fatte da Guarino, si veda Gualdo Rosa, «Le traduzioni latine...» cit., p.284, n.13 e ID., La fede nella paideia cit., p.24.

16. Plutarco, Vite di Plutarco, de gli huomini illustri greci et romani, Lodovico Domenichi (trad.), Venezia: Giolito, 1566-1568.

17. Per i testi originali, cito solo l'ultima edizione: [Homero], Homeric Hymns. Homeric Apocrypha. Lives of Homer, Martin L. West (ed.), Cambridge, Mass.; London: Harvard 
cronologia e vita di Omero, ma Jenson l'eliminò, per cui Palencia non la tradusse. Questi due opuscoli ebbero tanta fortuna da risultare inclusi nell'editio princeps delle opere di Omero a cura di Demetrio Calcondida nel 1488, insieme con gli Inni omerici, la Batracomiomachia e il Discorso su Omero di Dione Crisostomo.

Nella traduzione di Palencia il testo è preceduto dalla rubrica seguente:

Plutarcho philosopho escriuio en griego la vida del ylustre varon Homero. Boluiola en latin Guarino Verones. Y el cronista Alfonso de Palençia la traduxo en romançe castellano

Palencia, Plutarco, II, f.322v.

Queste parole non facevano altro che rispecchiare il titolo originale dell'edizione usata: «Homeri viri illustris vita ex Plutarcho graeco in latinum per Guarinum Veronensem versa» (ed. 1478, f. 458v.). Quindi, il Palentino non nutriva alcun sospetto su un testo falsamente attribuito a Plutarco e su una traduzione erroneamente aggiudicata a Guarino.

\section{Senofonte e l'Agesilao, tradotto da Battista Guarino}

Una delle biografie all'interno della vulgata latina delle Vite parallele corrispondente ad Agesilao, messo a confronto con Pompeo, è in realtà opera non di Plutarco ma di Senofonte (Atene, ca. 430 - Corinto, ca. 354). Per di più, nell'editio princeps e nelle successive, la versione era considerata frutto di Antonio Pacini, mentre il vero traduttore era Battista Guarini, figlio di Guarino, che intorno al 1459 l'aveva dedicata ad Ermolao Barbaro. Esisteva un'altra traduzione del testo di Senofonte portata a termine nel 1432 da Francesco Filelfo e dedicata al cardinale Niccolò Albergati. Nel frattempo, il testo plutarcheo con la biografia del generale spartano era stato tradotto il 28 ottobre 1462 e inviato a Lorenzo de' Medici. ${ }^{18}$ Dunque, Campano non incluse la pur esistente traduzione del testo plutarcheo sia perché i suoi scrupoli personali lo spingevano a propendere verso un testo più encomiastico (com'era lo scritto di Senofonte), sia perché, una volta capitatagli tra le mani, la credette opera autentica di Plutarco, cadendo in un grave errore. ${ }^{19}$

Di conseguenza, Alfonso de Palencia tradusse il testo di Senofonte senza sospettare che il testo fosse stato scambiato, visto che traduceva dal latino senza sapere il greco e l'errore proveniva dall'edizione utilizzata.

University Press, 2003. Per questa traduzione fatta da Pellegrino degli Agli, si veda il mio contributo: Susanna Allés Torrent, «Le vite di Omero tradotte da Pellegrino degli Agli», in Coesistenza e cooperazione nel Medioevo, Turnhout: Brepols, 2013 (in stampa).

18. Per quanto riguarda il contesto della traduzione, si veda PADE, The Reception, cit., p. 334-335; GiUstiniani, Sulle traduzioni, cit. p. 33.

19. Questa sembra essere l'ipotesi di Giustiniani: «Questo scritto di Senofonte è stato evidentemente scambiato per quello di Plutarco dall'ugual titolo e lo sostituisce nella raccolta» (Sulle traduzioni, cit. p. 33). 


\subsection{Ricezione di lavori biografici nella Spagna del secolo XV}

Ci furono inoltre - e dal mio punto di vista costituisce il gruppo più interessante- opere originali scritte da umanisti a imitazione delle Vite plutarchee.

\section{Cicero novus di Leonardo Bruni}

Leonardo Bruni, autore di grande influsso nella Penisola Iberica, scrisse una biografia di Cicerone, che spazia dalla traduzione dell'omonima vita plutarchea alla rielaborazione libera, poiché modificò sostanzialmente il testo e aggiunse informazioni ricavate da altre fonti. Ma fu soprattutto l'immagine di Cicerone ad essere cambiata da Bruni: a suo dire, la biografia di Plutarco non rendeva la vera personalità dell'oratore, non ne rispecchiava il ruolo politico svolto per la libertà repubblicana né la posizione cardinale come precettore di retorica ed oratoria e come esempio di lingua pura e stile elegante.

Bruni iniziò il suo lavoro, oggi conosciuto come il Cicero Novus, verso il 1413 e lo terminò nel 1415. ${ }^{20}$ Lo stesso Bruni, nella prefazione dell'opera rivolta a Niccolò Niccoli, racconta di aver trovato una traduzione della biografia plutarchea di Cicerone, probabilmente quella di Iacopo Angeli (13601410/1411). Oltre di ritenerla una versione di scarso valore, accusava il testo plutarcheo di aver silenziato una serie di episodi, per cui non offriva il meritato omaggio al rettore. Perciò, decise di omettere sia il testo plutarcheo che la traduzione pervenutagli. ${ }^{21}$

La fortuna del Cicero nouus fu molto larga e, come scrive Monfasani, il suo influsso si nota in autori come Giorgio da Trebisonda e nei suoi primi scritti, per esempio il De laudibus Ciceronis (1412), in cui si staglia il ritratto bruniano di un Cicerone eroe dell'umanesimo civile rappresentante dell'eloquenza e della dottrina di condottiero politico. ${ }^{22}$ Ma soprattutto la sua fortuna si agganciò al corpus latino plutarcheo fin dai suoi inizi poiché, una volta inserita

20. L'opera è stata edita in diverse occasioni, cito solo: Leonardo BrUni, Opere letterarie e politiche, Paolo VITI (ed. e trad. a cura di), Torino: UTET, 1996, p. 411-499, dove si data il lavoro il 18 ottobre 1415 (Ibid., p. 413); d'altro canto, PADE, The Reception, cit. I, p. 154-165 retrodata la stesura del testo al 1413. Ricorderò tre studi indispensabili: Edmund B. Fryde, «The Beginnings of Italian Humanist Historiography: The 'New Cicero' of Leonardo Bruni», English Historical Review, n. 95, 1980, p. 533-552; ID., Humanism and Renaissance Historiography, London: Hambledon Press, 1983, p. 33-54; Gary Ianziti, «A Life in Politics: Leonardo Bruni's Cicero", Journal of the History of Ideas, 61, 1, Jan. 2000, p. 39-58.

21. Così si esprime: «Nos igitur et Plutarcho et eius interpretatione omissis, ex iis que vel apud nostros vel apud Grecos de Cicerone scripta legeramus, ab alio exorsi principio vitam et mores et res gestas eius maturiore digestione et pleniore notitia, non ut interpretes sed pro nostro arbitrio voluntateque, descripsimus. Est autem nihil a nobis temere in historia positum, sed ita ut de singulis rationem reddere et certa probatione asserere valeamus» (Leonardo Bruni, Sulla perfetta traduzione, Paolo Viti (ed. e trad. a cura di), Napoli: Liguori, 2004, p. 249-250.

22. John Monfasani, George of Trebizond. A Biography and Study of his Rhetoric and Logic, Leiden: E.J. Brill, 1976, p.42. 
nell'editio princeps romana, sostituì la vita autentica scritta da Plutarco e fu a lungo scambiata per un originale dello scrittore greco. La situazione si prolungò fino al 1514, anno in cui riapparse nelle vulgate latine del corpus la traduzione di Iacopo Angeli dell'originale plutarcheo, seppure sotto il nome erroneo di Achille Bocci (1488-1562). 23

Conserviamo la traduzione spagnola di Alfonso de Palencia che la tradusse considerandola un'opera di Plutarco e ritenendo che l'aretino ne fosse il traduttore:

Plutarcho philosopho escriuio en griego la vida del ylustre varon Marco Tulio. Boluiola en latin Leonardo Aretino. Y el cronista Alfonso de Palençia la traduxo en romançe castellano.

Palencia, Plutarco, I, f.213vv.

\section{La Vita Aristotelis di Leonardo Bruni}

Bruni terminò la Vita Aristotelis prima del 13 aprile 1430, data in cui scrive al cardinale Niccolò Alberghati ringraziandolo per le sue buone parole a proposito dell'opera. ${ }^{24}$ Bruni aveva in mente di ripristinare l'immagine di un Aristotele uomo, sottolineandone la humanitas e distanziandolo dalla figura di filosofo della tradizione scolastica medievale. Il suo sforzo è compiuto con un nuovo metodo storiografico che nulla ha a che vedere con i precetti dell'età anteriore, in particolar modo per l'uso critico delle fonti.

Bruni era stato un grande traduttore di Aristotele, con un'attenzione specifica rivolta alla sua filosofia pratica, e in primis all'Etica e la Politica. Due immagini diverse del filosofo greco si sovrapponevano: l'Aristotele medievale, maestro di logica e dialettica, tanto familiare e amato quanto venerato nelle scuole, e un Aristotele in carne ed ossa, capace di offrire una filosofia pratica valida nel presente per la costruzione di un modello di vita civile.

La vita aveva circolato in forma manoscritta fino al 1470, anno in cui fu inclusa nella vulgata plutarchea, e da allora accompagnò tutte le ristampe, benché dal 1478 figurasse sotto il nome di Guarino Veronese.

23. Giustiniani, Sulle traduzioni, cit. p. 38.

24. Le edizioni più recenti della Vita Aristotelis sono: Bruni, Opere, cit. pp.501-530; e quella edita da Włodzimierz Olszzaniec in Lech Szczucki - Barbara Milewska-WaźbińsKa (a cura di), Humanistyczne żywoty filozofów starożytnych, Warszawa: Wydawnictwa Univwersytetu Warszawskiego, 2008, pp. 155-194. Alcuni studi interessanti: Jerrold E. Seigel, Rhetoric and Philosophy in Renaissance Humanism. The Union of Eloquence and Wisdom, Petrarch to Valla, Princeton (N.J.): Princeton University Press, 1968, p. 111-113; Edmund Fryde, «The first Humanistic Life of Aristotle: the Vita Aristotelis of Leonardo Bruni», in P. Denley-C. Elam (a cura di), Florence and Italy: Renaissance Studies in Honour of Nicolai Rubinstein, London: Westfield College. University of London. Committee for Medieval Studies, 1988, p. 285-96; Gary J. Ianziti, «Leonardo Bruni and biography: The Vita Aristotelis», Renaissance Quarterly, 55, 3, 2002, p. 805832. 
In Spagna, si ha notizia solo di una traduzione anonima appartenente al circolo del Marchese di Santillana, conservata nella BNE, ms. 10171, dipendente dal testo trasmesso dal codice magliabecchiano IX-2 della BNCF. ${ }^{25}$

Palencia sapeva che quest'opera era da attribuire a Leonardo Bruni, ed emendò l'errore presente nell'edizione da lui usata per portare a termine la sua versione:

Guarino Verones, segund aquesta collecçión latina, compuso la vida del ylustre varon Aristotele recogida de otros auctores. Y el cronista Alfonso de Palençia la traduxo de latin en romançe castellano. Mas la verdad es que la colligio Leonardo Aretino e la dirigio el cardenal de Sancta Cruz.

Palencia, Plutarco, II, f. 334 r.

Il dato non sorprende, vista la familiarità degli autori spagnoli con le opere di Bruni. Del resto, la traduzione del Palentino non comporta le connotazioni programmatiche che poteva avere fra gli umanisti italiani il ripristino della figura dello Stagirita per contrapposizione alla tradizione medievale, ma si tratta comunque di un episodio notevole nella storia della ricezione. Negli anni successivi e lungo il XVI secolo l'opera fu letta e riletta, come dimostrano alcuni riferimenti in autori come Pedro Mexia, ma studiarne le sorti ci porterebbe a un'analisi che va ben oltre i limiti di quest'articolo e che rimando ad un'altra sede.

\section{La Vita Platonis di Guarino Veronese}

A differenza di quanto accaduto con Leonardo Bruni, di Guarino Veronese si tradusse ben poco in terre ispaniche. ${ }^{26}$ Per limitarci al genero in questione, Guarino scrisse due biografie, una di sant'Ambrogio e un'altra di Platone, ${ }^{27}$ della quale conserviamo unicamente una traduzione in spagnolo ad opera di Alfonso de Palencia.

Guarino elaborò la Vita Platonis verso il 1430 e la dedicò al milanese Filippo di Giovanni Pellizzone, il medico del marchese Niccolò III di Ferrara, città in cui il Veronese risedeva sin dall'aprile $1429 .{ }^{28}$ Quindi, solo a distanza di un anno dalla stesura della Vita Aristotelis del Bruni, apparse una Vita che è stata da alcuni rimproverata per lo scarso valore critico nella scelta delle fonti, per l'uso a volte indiscriminato di Diogene Laerzio, e per l'approccio cristia-

25. Giuseppe Mazzocchi-Olga Perotti, «La "Vida de Aristóteles” di Bruni: edizione e studio», Cultura Neolatina 64, n. 1-2, 2004, p. 251-284.

26. Il «Progetto Boscan» registra di Guarino solo una traduzione del De linguae latinae differentis.

27. La biografia ambrosiana è contenuta in soli due manoscritti: Ferrara, Biblioteca comunale ariostesca, cod. II 90 e II 135; Cfr. Gino Pistilli, s.v. "Guarino Guarini», DBI 60, 2003, p. 367; della Vita Platonis abbiamo un'unica edizione in Tomasz PŁóciennik in Szczucki -MilewsKa-WaźBińsKa, Humanistyczne żywoty, cit., p. 195-278.

28. Marianne PAde, "Guarino and Caesar at the Court of the Este», in M. PAde-L. WAage Petersen-D. Quarta (a cura di), La corte di Ferrara e il suo mecenatismo 1441-1598, Copenhagen: Museum Tusculanum; Modena: Panini, 1990, p. 71-91. 
nizzante della figura platonica, riflesso della tradizione medievale agostiniana. ${ }^{29}$ $\mathrm{E}$ infatti, Platone è presentato come un cristiano avant la lettre, che avrebbe perfino conosciuto i profeti dell'autentica fede. Anche a Guarino interessava, anzitutto, sottolineare la humanitas del personaggio e l'attualità del suo pensiero nella vita civile del momento.

La Vita Platonis ebbe una fortuna relativa: conservata in almeno cinque manoscritti, ottenne numerose ristampe del corpus campaniano, ma non fu tradotta in lingua italiana fino al 1566, e grazie a Domenichi. ${ }^{30}$

La traduzione spagnola riproduce il grave problema testuale presente già nell'edizione di Campano, ma ha il merito di essere l'unica esistente. Nel preambolo si legge:

Guarino Verones escrivio en latin la vida del ylustre philosopho Platon colligida y compuesta por el. Y el cronista Alfonso de Palençia la traduxo en romançe castellano, después de escriptas e romançadas las vidas de Plutarcho.

Palencia, Plutarco, II, f.324v.

Ciò dimostra che Palencia sapeva chi era Guarino, che viene qualificato, nel prologo, come un "varón muy mentado en enseñança», con la caratteristica che lo distingueva dagli altri umanisti messa così in risalto. E seppure il gentilizio che accompagnava il nome esplicitava la sua patria, il Palentino non dubita a ostentare la propria cultura quando, trovandosi di fronte alla semplice frase inserita da Guarino a proposito di Catullo: «conterraneo meo Catulo», decide di trasformarla in "Catulo, mi conterraneo de Verona».

Come nel caso di Aristotele, non si può dire che le implicazioni di una tale traduzione siano le stesse che l'opera aveva avuto tra gli umanisti italiani, laddove autori come Hankins arrivano ad affermare che il Quattrocento italiano $\mathrm{fu}$ «un'epoca in cui la filosofia di Platone venne studiata e apprezzata più che in qualsiasi altra dopo la chiusura dell'Accademia di Atene, per ordine di Giustiniano, nel 529», ${ }^{31}$ ma le parole preliminari della traduzione indicano che Palencia aveva informazioni di prima mano rispetto al vero autore dell'opera.

$\mathrm{Ci}$ furono altre biografie platoniche. Una dovuta a Giovanni Tortelli (Arezzo 1400-1466), inserita nella sua De Orthographia e sotto la voce «Platone», si limita a una sintesi, a cui vanno aggiunti altri dati sparsi sotto le voci de "Anitos» e «Socrate»; un'altra — come non poteva essere altrimenti- fu originata dalla penna di Marsilio Ficino ed anteposta all'edizione dei Dialoghi platoni-

29. Nel descrivere il lavoro guariniano si è detto: «adattamento dalle Vite dei filosofi di Diogene Laerzio e da Agostino» (PIstilli, s.v. «Guarino Guarini», cit. p. 362) oppure: «Guarino tried to keep as closely as possible to the Lives and Opinions of Eminent Philosophers of Diogenes Laertius, using the lives of both Socrates and Plato» (Fryde, Humanism and Renaissance Historiography, cit., p. 63).

30. Per i manoscritti si veda, Szczucki-Milewska-Waźbińska, Humanistyczne żywoty, cit., p. 200. Per le ristampe, si veda: Giustiniani, Sulle traduzioni, cit., p.42.

31. James Hankins, La riscoperta di Platone nel Rinascimento italiano, S. U. BaldassariD. Downey (ed. e trad, a cura di), Pisa: Scuola Normale Superiore, 2009, p. 34. 
ci in traduzione latina, ma era molto più incentrata su questioni filosofiche, e non tanto su problemi storici.

\section{La Vita Caroli Magni di Donato Acciaiuoli}

Uno dei grandi nomi dell'umanesimo fu senz'altro Donato Acciaiuoli, ma di lui — stando ai dati in mio possesso- non venne tradotta nessuna opera, benché se ne conoscesse benissimo la figura. Acciaiuoli scrisse tre biografie, di cui due in copia parallela allo stile di Plutarco: la Vita Caroli Magni (1460) e le Vitae Hannibalis et Scipionis (1467-78). Si tratta, insomma, di un capitolo della ricezione dell'umanesimo che non è stato ancora studiato attentamente.

In occasione di un suo primo viaggio in Francia per la coronazione di Luigi XI (1423-1483), Acciaiuoli elaborò «in segno di fede e dono» una vita di Carlomagno. ${ }^{32}$ Il fiorentino voleva guadagnarsi il favore del re francese attraverso il ricupero della figura di Carlomagno che veniva attualizzata secondo i principî morali, etici ed intellettuali degli umanisti, in quanto precettori di príncipi. Le fonti utilizzate furono con molta probabilità la vita omonima di Eghinardo, il Liber pontificalis, le croniche di Ricordano Malispini e di Giovanni Villani, e la Historia Florentini populi di Bruni, e l'ispirazione si rivolgeva verso lo stile e i passi di Plutarco, Cesare e Tito Livio. L'obiettivo era quello di proporre una revisione della figura di Carlomagno alla luce dei nuovi precetti storiografici umanistici.

La Vita Caroli Magni riscosse una considerevole fortuna e si diffuse manoscritta, anche e soprattutto grazie al curatore, Campano, che conosceva il testo sin dalla fine del 1467. ${ }^{33}$ La traduzione spagnola di Alfonso de Palencia è anche in questo caso l'unica esistente. Il traduttore conosceva l'autore, essendo questi correttamente situato col gentilizio nel titolo della versione:

Donato Acciolo, çibdadano florentino, escrivio en latin la vida del ylustre varon Carlo Magno, recolegida de las historias, y el cronista Alfonso de Palencia la traduxo en romançe castellano. Aquesta vida es mas moderna y de principe catolico y bien mereciente de la religion cristiana

Palencia, Plutarco, II, f. 338r

Insomma, Palencia era consapevole del fatto che Acciaiuoli aveva portato a termine un lavoro critico di fonti («recolegida de las historias»); identificava l'autore come «cibdadano florentino» e aggiungeva «de principe catolico y bien

32. La bibliografia sulla Vita Caroli Magni è relativamente scarsa; per una visione d'insieme, rimando al mio contributo: S. Allés Torrent, "The Vita Caroli Magni of Donato Acciaiuoli, translated by Alfonso de Palencia», in B. TAYlor-A. Coroleu (a cura di), Humanism and Christian Letters in Early Modern Iberia (1480-1630), Newcastle: Cambridge Scholars Publishing, 2010, p. 61-74.

33. Campano, infatti, conosceva di prima mano l'opera di Acciaiuoli, così si legge in una sua lettera pubblicata in Iacopo Ammannati Piccolomini, Lettere (1444-1479), Paolo Cherubini (a cura di), Roma: Pubblicazioni degli Archivi di Stato, 1997, vol. II, p. 625: Ep. 251. Per la circolazione manoscritta si veda, KRISTELler, Iter, cit., che ne arriva a elencare undici testimoni: Italia (9), Polonia (1), Stati Uniti (1). 
mereciente de la religion cristiana», a dimostrazione che la scarsa adeguazione di un personaggio estraneo alla tipologia plutarchea non era passata inavvertita.

\section{Le Vitae Hannibalis et Scipionis di Donato Acciaiuoli}

Posteriormente, Acciaiuoli intraprese la stesura di due biografie di personaggi confrontati, Annibale e Scipione, terminate il 15 giugno 1467 e dedicate a Piero de'Medici. ${ }^{34}$ Acciaiuoli elaborò perfino la synkrisis finale, caratteristica delle Vite parallele di Plutarco, paragonando i due personaggi e, benché si mostri sempre favorevole a Scipione quale modello di coraggio e virtù, l'approccio critico cerca di bilanciare il carattere e le azioni dei due generali. La confusione tra le vite omonime ipoteticamente scritte da Plutarco e quelle dell'Acciaiuoli fu così grave che, collocate in modo strategico nel posto cronologicamente giusto all'interno del corpus latino edito da Campano, per molti secoli furono ritenute opere autentiche del Cheronese. ${ }^{35}$

Queste vite, oltre ad avere una modesta circolazione manoscritta, accompagnarono sempre le versioni latine delle Vite parallele di Plutarco. Di conseguenza, le due biografie di Annibale e Scipione furono tradotte in spagnolo da Alfonso de Palencia, il quale — se si tiene conto dei suoi interessi storiografici- doveva provare un grande piacere nella lettura grazie alla ricchezza delle fonti utilizzate (Polibio, Tito Livio, Plutarco, Nepote, Giustino).

La versione spagnola fu preceduta solamente da quella italiana di Battista Alessandro Jaconello, stampata all'Aquilla il 16 settembre $1482 ; ;^{36}$ la successiva traduzione italiana non vide la luce fino al 1566 per mano del già menzionato Domenichi. E tutti credevano di aver tradotto e di tradurre un'opera originale di Plutarco. Così lo indica un passo molto significativo della versione di Alfonso di Palencia, in cui Acciaiuoli cita direttamente, tra altri autori, Plutarco: «Nam Polibius Plutarchus et alii locupletissimi auctores Corneliam: quae Caium et Tyberium peperit: post mortem Aphricani Graeccho nupsisse scribunt»

34. La data figura nel manoscritto Napoli, Biblioteca Nazionale, XIII A A 19: «Donatus Acciaiolus, vitae Hannibalis et Scipionis», dedicata a "Petri Medices» e come colofone: «Publii Cornelii Scipionis vita a Donato Acciaiolo Florentino edita XV Junii MCCCCLXVII explicit. Transcripsit Florentiae hoc exemplar P. Cenninus XXVI Januar 1468» (f. 65v) (Kristeller, Iter, vol. II, «Italy», 1998, p. 547). Esistono pochi studi sul tema, tra i quali: Barbara SCARDigLI, "C’è qualcosa di plutarcheo nella vita di Scipione dell'Acciaiuoli?», in I. Gallo, L'eredità culturale di Plutarco dall'antichità al Rinascimento, Napoli: D’Auria, 1998, p. 289-297; Monica Affortunati-Barbara Scardigli, «La vita "plutarchea" di Annibale. Un'imitazione di Donato Acciaiuoli», A\&R, 37, 1992, p. 88-150.

35. Il caso più interessante è l'edizione di un'opera sotto questo titolo: Annibale e Scipione: dalle Vite parallele di Plutarco / con introduzione e note di Avancinio Avancini, Milano: Vallardi, 1924.

36. Plutarco, Vitae Parallelae, Thesei, Romuli, Lycurgi, Numae, Themistoclis, Camilli, Hannibalis, Scipionis, timoleonis, Aemilii Pauli, Pyrri, Lysandris, Scillae, Eumenis, Sertorii, Cimonis, Luculli, Niciae, Crassi, Agesilai, Pompei, Alexandri, Caesaris, Phocionis, Catonis. Battista Alessandro Jaconello (trad. it.). Facsimile della edizione aquilana del Rotweil stampata nel 1482, L'Aquila: Comtitato per il $5^{\circ}$ Centenario della introduzione della stampa in Abruzzo, 1982. 
(ed. 1478, f. 129r). Ebbene, Palencia pensò che la citazione del nome del Cheronese fosse un errore, cancellandolo così dall'elenco: «Ca Polibio e otros muy ricos auctores escriven que Cornelia la que parió a Gayo e a Tiberio fue casada con Graccho después de la muerte del Africano» (Palencia, Plutarco, II, f.191r).

\section{Conclusioni e ridimensionamento della ricezione del genero biografico in Spagna}

Come ho cercato di dimostrare seppur per sommi capi, la serie di lavori elencati ha un interesse molto ampio per più di un motivo. Innanzitutto, le traduzioni delle tre opere greche, che indirettamente sono arrivate nella Penisola Iberica, sottolineano oltremodo il ruolo cruciale dell'umanesimo italiano nel recupero dei testi classici; così l'Evagora di Isocrate, l'Agesilao di Senofonte, e il De Homero di Pseudo-Plutarco trovarono un'eco in terre castigliane, anche se - come ho illustrato- il traduttore molto probabilmente non immaginava che l'autore tradotto non fosse Plutarco. La vita di Attico di Cornelio Nepote destò alcuni sospetti, per cui venne ipotizzata una successione di fatti poco credibili: la biografia di Attico sarebbe stata scritta in latino da Nepote e successivamente tradotta dal latino al greco dallo stesso Plutarco. Comunque sia, il fatto è che Palencia collocava correttamente i due autori entro le loro coordinate cronologiche.

Il capitolo più interessante e sul quale ho incentrato più sforzi è costituito dalle biografie originali scritte da umanisti di primo livello, quali Leonardo Bruni, Guarino Veronese e Donato Acciaiuoli. E lo è essenzialmente per due ragioni. Da un lato, queste traduzioni si affiancano alle opere inizialmente menzionate, e più largamente studiate dalla critica, cioè la Vita Socratis et Senecae di Giannozzo Manetti, letta ma non tradotta in Spagna, e la Vita Homeri elaborata da Pier Candido Decembrio, esistente in una versione castigliana anonima. Le versioni castigliane di Palencia della Vita Aristotelis, la Vita Platonis, la Vita Caroli Magni (malgrado che questa cronologicamente si separi dalle altre) e le Vitae Hannibalis et Scipionis rappresentano, come dimostrano le ricerche altrove da me intraprese ${ }^{37}$, un arricchimento notevole in questo mare di traduzioni quattrocentesche. Nel caso di Bruni si conferma l'ampio interesse suscitato dalla sua figura nella Penisola Iberica; in quello di Guarino, si affianca alla traduzione castigliana anonima e parziale del De Lingue Latine Differentiis una seconda traduzione della sua vita platonica; infine, nel caso dell'Acciaiuoli le biografie costituiscono le sue uniche opere tradotte in spagnolo. D'altro canto, i testi ravvivano tradizioni fecondissime di autori e aiutano a tracciare in maniera più precisa la fortuna in territorio ispanico di grandi autori e figure storiche, come Platone, Aristotele, Scipione, Annibale o Carlomagno.

37. Susanna Allés Torrent, Parallelae sive Vitae illustrium virorum (Las vidas de Plutarco, Sevilla 1491). Estudio y edición crítica de la traducción de Alfonso de Palencia de las vidas compuestas por Donato Acciaiuoli, Leonardo Bruni y Guarino Veronese [Tesi di dottorato], Universitat de Barcelona, 2013. 